\title{
THE NON-ISOTROPIC DIFFUSION APPROXIMATION IN WOLF-RAYET WINDS
}

\author{
KENNETH G. GAYLEY AND STANLEY P. OWOCKI \\ Bartol Research Institute, University of Delaware, Newark DE 19716, USA
}

\begin{abstract}
We derive the dynamics of a steady-state Wolf-Rayet wind using a non-isotropic diffusion approximation applied to the transfer between strongly overlapping spectral lines. Following the approach of Friend and Castor (1983), the line list is assumed to approximate a statistically parametrized Poisson distribution in frequency, so that photon transport is controlled by an angle-dependent, effectively gray opacity. We find that multiple radiative momentum deposition is greatest when photons undergo a nearly local diffusion, e.g. through scattering between many lines closely spaced in frequency. Our results reiterate the view that the so-calied "momentum problem" of WR winds is better characterized as an "opacity problem" of simply identifying enough driving lines. One way of increasing the number of thick lines in WR winds is to transfer opacity from saturated to unsaturated lines, yielding a steeper opacity distribution than that found in OB winds. We also consider the growth of instabilities, and find that WR winds are up to an order of magnitude less unstable than $\mathrm{OB}$ winds if both are driven radiatively. However, initially small perturbations can still be multiplied by many orders of magnitude as they advect through WR winds.
\end{abstract}

\section{The Second-Order NID Approximation}

We first apply the effectively gray opacity model of Friend and Castor (1983) to establish an overall opacity scale $\tau$, and then write the radiative transfer equation for the mean radiation field in spherical coordinates. This is

$$
I_{\mu}=S(\mu)+\frac{\mu}{f(\mu)} \frac{\partial}{\partial \tau} I_{\mu}-\frac{\left(1-\mu^{2}\right)}{f(\mu)} \frac{\lambda}{r} \frac{\partial}{\partial \mu} I_{\mu},
$$

where $f(\mu)$ gives the angular dependence of the effectively gray opacity (where $f[1]=1$ ), and $\lambda$ is the mean free path between spectral line encounters for radial photons.

Equation (1) can be used to generate an infinite sum of derivatives of $S(\mu)$ by successively substituting the expression for $I_{\mu}$ into every appearance of $I_{\mu}$ on the right-hand side. The objective in a diffusion approximation is to treat these derivatives as small so that the sum may be truncated to some order. In order to derive a non-zero instability, it is necessary to carry this sum to second order in the quantity $\lambda / r$. Of course, this approach will only be valid if $\lambda / r<<1$, which is the limit of extreme line overlap. The second-order expansion gives that the desired angular dependence of $I_{\mu}$ can be written as 


$$
\begin{gathered}
I_{\mu}^{N I D}-S(\mu)=\left\{\frac{\mu}{f(\mu)}-\right. \\
\left.\frac{\left(1-\mu^{2}\right)}{f(\mu)^{2}} \frac{\lambda}{r}\left[1-\frac{\partial \ln f(\mu)}{\partial \ln \mu}\right]-\frac{\mu^{2}}{f(\mu)^{2}} \frac{\lambda}{r} \frac{\partial \ln f(\mu)}{\partial \ln r}\right\} S^{\prime}+\frac{\mu^{2}}{f(\mu)^{2}} S^{\prime \prime}
\end{gathered}
$$

where $S^{\prime}=\partial S / \partial \tau$ and $S^{\prime \prime}=\partial^{2} S / \partial \tau^{2}$ are $\mu$ independent to this order.

Evaluating $S^{\prime}$ and $S^{\prime \prime}$ by requiring a constant luminosity and assuming effectively gray radiative equilibrium, and approximating the derivatives of $f(\mu)$, we obtain the expression for $I_{\mu}$, which simplifies at the point of isotropic expansion (a characteristic point in the wind) to

$$
I_{\mu}^{N I D} \cong S+3 \mu H-3 H \frac{\lambda}{r}\left[1-3 \mu^{2}+\mu^{2}\left(\frac{9}{5}-3 \mu^{2}\right) \alpha \frac{\kappa_{r}}{\kappa_{e s}+\kappa_{r}}\right]
$$

where $H=L_{*} / 16 \pi r^{2}$ is the astrophysical flux, $\alpha$ is the standard power-law parameter for the line distribution (Castor et al. 1975), and $\kappa_{e s}$ and $\kappa_{r}$ are the free electron and radial effectively gray line cross sections per gram.

This approximation for the mean intensity allows us to find the growth rate of small-scale radial velocity perturbations, evaluated at the radius of isotropic expansion for simplicity. The result is $\partial g / \partial v=\Omega g / v_{t h}$, where

$$
\Omega \cong \frac{8}{5} x_{\alpha}\left(1+\frac{9}{35} \alpha \frac{\kappa_{r}}{\kappa_{e s}+\kappa_{r}}\right)\left(\frac{\lambda}{r}\right) \cong 1.8 x_{\alpha}\left(\frac{\lambda}{r}\right)
$$

is essentially the fractional growth over a Sobolev length, and $x_{\alpha}$ ranges from 0.5 to 1 as $\alpha$ varies from 0.5 to 0.7 . We note further that $\lambda / r$ is of order the inverse of the momentum efficiency factor $P_{\text {wind }}=\dot{M} v_{\infty} c / L_{*}$, so the growth rate is suppressed in high mass-loss winds. However, the number of e-folds over the extent of the wind, denoted $N_{\text {fold }}$, is still large, as seen in Table 1 for a unitless mass loss rate of $10^{3}$.

Table 1. Integrated Wind Attributes for $\frac{\dot{M}}{L_{*} / c^{2}}=10^{3}$

\begin{tabular}{lllll}
\hline$\alpha$ & $\Gamma$ & $N_{\text {fold }}$ & $\tau_{\text {tot }}(\mu=1)$ & $P_{\text {wind }}$ \\
\hline .5 & .7 & 22 & 12 & 7 \\
.5 & .5 & 35 & 13 & 9 \\
.75 & .7 & 130 & 17 & 30 \\
.75 & .5 & 230 & 24 & 40 \\
\hline
\end{tabular}

\section{References}

Castor, J.I., Abbott, D.C., Klein, R.I. 1975, $A p J$ 195, 157

Friend, D.B., Castor, J.I. 1983, Ap.J 272, 259 